\title{
Sublimation temperature of circumstellar dust particles and its importance for dust ring formation
}

\author{
Hiroshi Kobayashi ${ }^{1}$, Hiroshi Kimura ${ }^{2}$, Sei-ichiro Watanabe ${ }^{3}$, Tetsuo Yamamoto ${ }^{4}$, and Sebastian Müller ${ }^{1}$ \\ ${ }^{1}$ Astrophysical Institute and University Observatory, Friedrich Schiller University Jena, Schillergaesschen 2-3, 07745 Jena, Germany \\ ${ }^{2}$ Center for Planetary Science, clo Integrated Research Center of Kobe University, \\ Chuo-ku Minatojima Minamimachi 7-1-48, Kobe 650-0047, Japan \\ ${ }^{3}$ Department of Earth and Planetary Sciences, Graduate School of Environmental Studies, Nagoya University, \\ Furo-cho, Chikusa-ku, Nagoya 464-8601, Japan \\ ${ }^{4}$ Institute of Low Temperature Science, Hokkaido University, Kita-Ku Kita 19 Nishi 8, Sapporo 060-0819, Japan
}

(Received November 10, 2010; Accepted March 10, 2011; Online published February 2, 2012)

\begin{abstract}
Dust particles in orbit around a star drift toward the central star by the Poynting-Robertson effect and pile up by sublimation. We analytically derive the pile-up magnitude, adopting a simple model for optical cross sections. As a result, we find that the sublimation temperature of drifting dust particles plays the most important role in the pile-up rather than their optical property does. Dust particles with high sublimation temperature form a significant dust ring, which could be found in the vicinity of the sun through in-situ spacecraft measurements. While the existence of such a ring in a debris disk could not be identified in the spectral energy distribution (SED), the size of a dust-free zone shapes the SED. Since we analytically obtain the location and temperature of sublimation, these analytical formulae are useful to find such sublimation evidences.
\end{abstract}

Key words: Sublimation, dust, interplanetary medium, debris disks, celestial mechanics.

\section{Introduction}

Refractory dust grains in orbit around a star spiral into the star by the Poynting-Robertson drag (hereafter P-R drag) and sublime in the immediate vicinity of the star. Because the particles lose their mass during sublimation, the ratio $\beta$ of radiation pressure to gravity of the star acting on each particle ordinarily increases. As a result, their radial-drift rates decrease and the particles pile up at the outer edge of their sublimation zone (e.g., Mukai and Yamamoto, 1979; Burns et al., 1979). This is a mechanism to form a dust ring proposed by Belton (1966) as an accumulation of interplanetary dust grains at their sublimation zone. Ring formation of drifting dust particles is not limited to refractory grains around the sun but it also takes place for icy grains from the Edgeworth-Kuiper belt and for dust in debris disks (Kobayashi et al., 2008, 2010). Therefore, dust ring formation due to sublimation of dust particles is a common process for radially drifting particles by the P-R drag.

The orbital eccentricity and semimajor axis of a dust particle evolve by sublimation due to an increase in its $\beta$ ratio as well as by the P-R drag. We have derived the secular evolution rates of the orbital elements (Kobayashi et al., 2009). The derived rates allow us to find an analytical solution of the enhancement factors for the number density and optical depth of dust particles due to a pile-up caused by sublimation. Our analytical solution is found to reproduce numerical simulations of the pile-up well but its applicability is

Copyright (c) The Society of Geomagnetism and Earth, Planetary and Space Sciences (SGEPSS); The Seismological Society of Japan; The Volcanological Society of Japan; The Geodetic Society of Japan; The Japanese Society for Planetary Sciences; TERRAPUB.

doi:10.5047/eps.2011.03.012 restricted for low eccentricities of subliming dust particles. The analytical solution shows that the enhancement factors depend on dust shapes and materials as expected from previous numerical studies (cf. Kimura et al., 1997). Although the solution includes physical quantities for the shapes and materials, it does not explicitly show which quantity essentially determines the enhancement factors.

The goal of this paper is to derive simplified formulae that explicitly indicate the dependence of dust ring formation on materials and structures of dust particles. In this paper, we adopt a simple model for the optical cross sections of fractal dust particles and analytically obtain not only the enhancement factors but also the location of the pile-up and sublimation temperature. In addition, we extend the model of Kobayashi et al. (2009) by taking into account orbital eccentricities of subliming dust particles.

In Section 2, we derive the sublimation temperature as a function of the latent heat. In Section 3, we introduce the characteristic radius of fractal dust and derive the sublimation distance for that dust. In Section 4, we simplify the formulae of enhancement factors derived by Kobayashi et al. (2009) and obtain the new formulae that show explicitly the dependence on materials and structures of the particles. We provide a recipe to use our analytical formulae in Section 5, apply our simplified formulae to both the solar system and extrasolar debris disks, and discuss observational possibilities of dust sublimation in Section 6. We summarize our findings in Section 7.

\section{Sublimation Temperature}

We consider dust particles in orbit around a central star with mass $M_{*}$. Driven by the P-R drag, they drift inward 
Table 1. Material parameters: the material density, $\mu$ is the mean molecular weight, $H$ is the latent heat of sublimation, and $P_{0}$ is the saturated vapor pressure $P_{\mathrm{v}}$ in the limit of high temperature.

\begin{tabular}{lcccc}
\hline & material density $\left[\mathrm{g} \mathrm{cm}^{-3}\right]$ & $\mu$ & $H\left[\mathrm{erg} \mathrm{g}^{-1}\right]$ & $P_{0}\left[\mathrm{dyn} \mathrm{cm}^{-2}\right]$ \\
\hline olivine & 3.3 & 169.1 & $3.21 \times 10^{10}$ & $6.72 \times 10^{14}$ \\
pyroxene & 3.3 & 60.1 & $9.60 \times 10^{10}$ & $3.12 \times 10^{11}$ \\
obsidian & 2.37 & 67.0 & $7.12 \times 10^{10}$ & $1.07 \times 10^{14}$ \\
carbon & 1.95 & 12.0 & $7.27 \times 10^{11}$ & $4.31 \times 10^{16}$ \\
iron & 7.86 & 55.8 & $2.97 \times 10^{10 \mathrm{a}}$ & $5.00 \times 10^{4 \mathrm{a}}$ \\
pure ice & 1.0 & 18 & $2.83 \times 10^{10 \mathrm{~b}}$ & $3.08 \times 10^{13 \mathrm{~b}}$ \\
dirty ice & 1.4 & 18 & $2.83 \times 10^{10 \mathrm{~b}}$ & $2.67 \times 10^{13 \mathrm{~b}}$ \\
\hline
\end{tabular}

Note-obsidian is formed as an igneous rock and may not be plausible as interplanetary dust, but is applied for a comparison with previous studies (e.g., Mukai and Yamamoto, 1979).

a,b $H$ and $P_{0}$ are obtained from the following formulae with the sublimation temperature of each material.

a $P_{\mathrm{v}}=1.33 \times 10^{4} \exp (-2108 / T+16.89-2.14 \ln T) \mathrm{dyn} \mathrm{cm}^{-2}($ Lamy, 1974).

${ }^{\mathrm{b}} \log P_{\mathrm{v}}=-2445.5646 / T+8.2312 \log T-0.01677006 T+1.20514 \times 10^{-5} T^{2}-3.63227\left(P_{\mathrm{v}}\right.$ in the cgs unit; Washburn, 1928)

until they actively sublime in the vicinity of the star. We have shown in Kobayashi et al. (2009) that the ring formation due to sublimation occurs only for their low orbital eccentricities $e$ and obtained the secular change of semimajor axis $a$ of the particle with mass $m$ as

$$
\left\langle\frac{d a}{d t}\right\rangle=-\left.\eta \frac{\beta}{1-\beta} \frac{a}{m} \frac{d m}{d t}\right|_{r=a}-\frac{\beta G M_{*}}{c} \frac{2}{a},
$$

where $\eta \equiv-\ln \beta / \ln m,-d m /\left.d t\right|_{r=a}$ is the mass-loss rate of the particle at the distance $r=a, G$ is the gravitational constant, and $c$ is the speed of light. The $\beta$ ratio is given by

$$
\beta=\frac{L_{*} \bar{C}_{\mathrm{pr}}}{4 \pi c G M_{*} m},
$$

where $\bar{C}_{\text {pr }}$ is the radiation pressure cross section averaged over the stellar radiation spectrum and $L_{*}$ is the stellar luminosity. The first and second terms on the right-hand side of Eq. (1) represent the drift rates due to sublimation and the P-R drag, respectively. Although we consider only the $\mathrm{P}-\mathrm{R}$ drag from stellar radiation, the P-R drag due to the stellar wind also transports the particles. However, the magnitude of pile-up, its location, and sublimation temperature hardly depend on which drag determines their transport (Kobayashi et al., 2008, 2009).

A particle generated in a dust source initially spirals toward a star by the P-R effect. As it approaches the star due to the P-R inward drift, its temperature rises high and it finally starts active sublimation. The drift turns outward by sublimation when $\beta$ increases with mass loss. The radial motion of the particles becomes much slower than the P$\mathrm{R}$ drift alone, resulting in a pile-up of the particles. Note that other mass-loss mechanisms such as sputtering by stellar winds and UV radiation are negligible during active sublimation. ${ }^{1}$

\footnotetext{
${ }^{1}$ We consider dust particles that can drift into their active sublimation zone. This is valid in the solar system, since the size decreasing timescale due to sputtering is longer than the drift time due to the P-R effect (Mukai and Schwehm, 1981). However, we note that icy particles may not come to their sublimation zone around highly luminous stars because of strong UV sputtering (Grigorieva et al., 2007).
}

The mass loss rate of a particle due to sublimation is given by

$$
-\frac{d m}{d t}=A \sqrt{\frac{\mu m_{\mathrm{u}}}{2 \pi k T}} P_{0}(T) \exp \left(-\frac{\mu m_{\mathrm{u}} H}{k T}\right),
$$

where $A$ is the surface area of the particle, $H$ is the latent heat of sublimation, $\mu$ is the mean molecular weight of the dust material, $m_{\mathrm{u}}$ is the atomic mass unit, and $k$ is the Boltzmann constant. Here the saturated vapor pressure at temperature $T$ is expressed by $P_{0}(T) \exp \left(-\mu m_{\mathrm{u}} H / k T\right)$ with $P_{0}(T)$ being only weakly dependent on $T$.

During active sublimation, the first term on the righthand side of Eq. (1) increases and then $\langle\dot{a}\rangle$ nearly vanishes. The temperature $T_{\text {sub }}$ at active sublimation is approximately determined by $\langle\dot{a}\rangle=0$. Substituting Eq. (3) into Eq. (1) for $\langle\dot{a}\rangle=0$, we have

$T_{\text {sub }}=-\frac{\mu m_{\mathrm{u}} H}{k}\left[\ln \left(\frac{2 G M_{*}}{c a^{2}} \frac{m}{A P_{0}} \frac{1-\beta}{\eta} \sqrt{\frac{2 \pi k T_{\mathrm{sub}}}{\mu m_{\mathrm{u}}}}\right)\right]^{-1}$.

Although Eq. (4) is a function of $a$ as well as $T_{\text {sub }}$, the natural logarithmic function on the right-hand side has little sensitivity to $a$ and $T_{\text {sub }}$. Therefore, the sublimation temperature may be approximated by

$$
T_{\text {sub }} \simeq 1.3 \times 10^{3} \xi^{-1}\left(\frac{H}{3.2 \times 10^{10} \mathrm{erg} \mathrm{g}^{-1}}\right)\left(\frac{\mu}{170}\right) \mathrm{K},
$$

where

$$
\xi=1+0.02 \ln \left(\frac{P_{0}}{6.7 \times 10^{14} \mathrm{dyn} \mathrm{cm}^{-2}}\right) .
$$

Here, we set $m=1.1 \times 10^{-12} \mathrm{~g}, \beta=1 / 2, \eta=1 / 3$, $T_{\text {sub }}=1300 \mathrm{~K}$ and $a=15 R_{\odot}$ with the solar radius $R_{\odot}$ in the argument of the logarithmic function under the assumption of a spherical olivine dust particle around the sun; the other choice of these values does not change the result significantly because of the slowly-varying properties of the logarithmic function. 


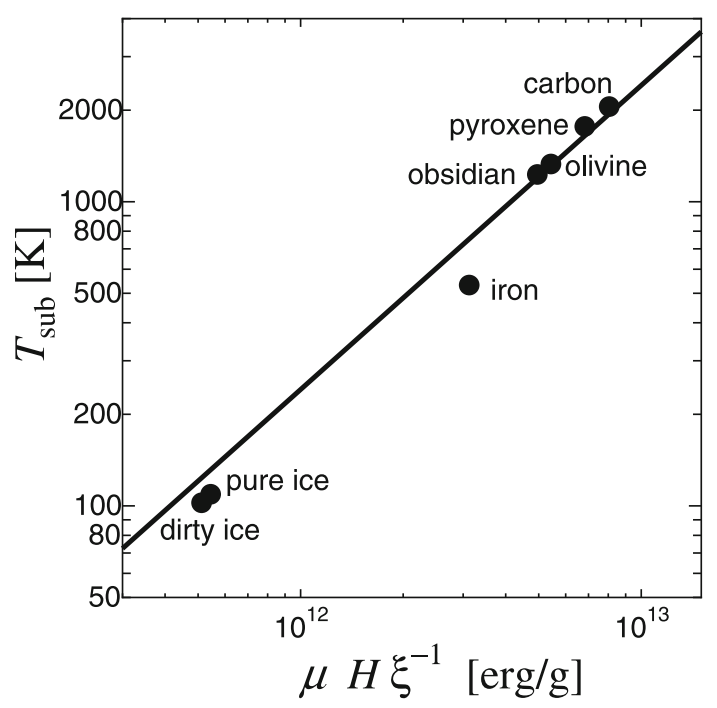

Fig. 1. Sublimation temperature $T_{\text {sub }}$ as a function of $\mu \mathrm{H}^{-1}$, where $\xi=1+0.02 \ln \left(P_{0} / 6.7 \times 10^{14} \mathrm{dyn}^{-2}\right)$. The mean molecular weight $\mu$, the latent heat $H$, and the vapor pressure in the limit of high temperature, $P_{0}$, are listed in Table 1. The solid line indicates Eq. (5). Circles represent $T_{\text {sub }}$ obtained from the method of Kobayashi et al. (2009) around the sun.

Equation (5) indicates that the active sublimation temperature is mainly determined by the latent heat of sublimation and mean molecular weight of the particles. This explains the findings by Kobayashi et al. (2008) that the temperature is insensitive to the stellar parameters, $M_{*}$ and $L_{*}$. As a consistency check, we calculate the sublimation temperatures according to Kobayashi et al. (2009) for materials listed in Table 1 and compare the temperatures with Eq. (5) (see Fig. 1). In spite of the simplification, Eq. (5) is in good agreement with the temperature given by the procedure of Kobayashi et al. (2009).

\section{Fractal Dust Approximation}

We introduce the characteristic radius $s$ of a dust particle, which is defined as

$$
s^{2}=\frac{5}{3} \frac{\int \rho_{\mathrm{i}} \tilde{s}^{2} d V}{\int \rho_{\mathrm{i}} d V},
$$

where $\int d V$ means an integration over volume, $\tilde{s}$ is the distance from its center of mass, and $\rho_{\mathrm{i}}$ is its interior density. We consider that particles have a fractal structure; the massradius relation of the particles is given by $m \propto s^{D}$ for a constant fractal dimension $D$. For the fractal dust, $s=s_{\mathrm{g}} \sqrt{5 / 3}$, where $s_{\mathrm{g}}$ is the gyration radius of the dust (Mukai et al., 1992). For homogeneous spherical dust, the characteristic radius reduces to the radius of the sphere. The cross sections of scattering and absorption of light are approximately described by a function of $\pi s^{2}$ and $2 \pi s / \lambda$, where $\lambda$ is the wavelength at the peak of light spectrum from the dust (Mukai et al., 1992).

The smallest dust particles before active sublimation contribute most to the enhancements of number density and optical depth at the pile-up (Kobayashi et al., 2009). Small particles produced by parent bodies in circular orbits are expelled by the radiation pressure if $\beta>1 / 2$. Thus, the minimum characteristic radius $s_{0 \text { min }}$ of dust particles prior to active sublimation corresponds to $\beta=1 / 2$. The radiation pressure cross section $\bar{C}_{\mathrm{pr}}$ is roughly given by $\pi s_{0 \text { min }}^{2}$ in Eq. (2) for $s_{0 \text { min }} \gtrsim \lambda_{*}$. Then, we have

$$
s_{0 \min }=1.2\left(\frac{L_{*}}{L_{\odot}}\right)\left(\frac{M_{\odot}}{M_{*}}\right)\left(\frac{\rho}{1.0 \mathrm{~g} \mathrm{~cm}^{-3}}\right)^{-1} \mu \mathrm{m},
$$

where $M_{\odot}$ and $L_{\odot}$, respectively, denote the solar mass and luminosity. Note that $\rho=3 m_{0 \min } / 4 \pi s_{0 \text { min }}^{3}$ is the effective density of a dust particle with the characteristic radius $s_{0 \text { min }}$ and mass $m_{0 \mathrm{~min}}$ in the following derivation. In addition, we discuss the application limit of our formulae in Appendix A.

\subsection{Sublimation distance}

We introduce the dimensionless parameter $x$,

$$
\begin{aligned}
x & =\frac{2 \pi s_{0 \mathrm{~min}}}{\lambda_{\text {sub }}} \\
& =3.4\left(\frac{L_{*}}{L_{\odot}}\right)\left(\frac{M_{\odot}}{M_{*}}\right)\left(\frac{\rho}{1.0 \mathrm{~g} \mathrm{~cm}^{-3}}\right)^{-1}\left(\frac{T_{\mathrm{sub}}}{1300 \mathrm{~K}}\right),
\end{aligned}
$$

where $\lambda_{\text {sub }}$ is the wavelength at the peak of thermal emission from subliming dust with temperature $T_{\text {sub }}$. We approximate $\lambda_{\text {sub }}=\left(2898 \mathrm{~K} / T_{\text {sub }}\right) \mu \mathrm{m}$, which is the wavelength at the peak of a blackbody radiation spectrum with $T_{\text {sub }}$.

Since we deal with dust dynamics in optically thin disks, the equilibrium temperature $T$ of a dust particle at a certain distance from a star is determined by energy balance among absorption of incident stellar radiation and emission of thermal radiation. Therefore, the relation between temperature $T$ and distance $r=a$ is approximately given by (e.g., Kobayashi et al., 2009)

$$
\frac{L_{*} \bar{C}_{*}}{4 \pi a^{2}}=4 \bar{C}_{\mathrm{d}} \sigma_{\mathrm{SB}} T^{4}
$$

if $a$ is much larger than the radius of the central star. Here, $\sigma_{\mathrm{SB}}$ is the Stephan-Boltzmann constant and $\bar{C}_{*}\left(s_{0 \mathrm{~min}}\right)$ and $\bar{C}_{\mathrm{d}}$ are the absorption cross sections integrated over the stellar spectrum and the thermal emission from the dust particle, respectively.

Because $s_{0 \text { min }}$ is larger than $\lambda_{*}$, the cross section $\bar{C}_{*}\left(s_{0 \text { min }}\right)$ is approximated by the geometrical cross section;

$$
\bar{C}_{*}\left(s_{0 \min }\right)=\pi s_{0 \min }^{2} .
$$

The cross section $\bar{C}_{\mathrm{d}}\left(s_{0 \text { min }}\right)$ may be $\pi s_{0 \min }^{2}$ for $x \gg 1$ and $\pi s_{0 \min }^{2} x$ for $x \ll 1$. We connect them in a simple form as

$$
\bar{C}_{\mathrm{d}}\left(s_{0 \min }\right)=\pi s_{0 \min }^{2}\left(\frac{x}{1+x}\right) .
$$

When the temperature of the smallest drifting particles reaches $T_{\text {sub }}$, their pile-up results in a peak on their radial distribution (Kobayashi et al., 2009). With the application of the cross sections given by Eqs. (11), (12) to Eq. (10), the sublimation distance $a_{\text {sub }}$ at the peak is obtained as

$$
a_{\mathrm{sub}} \simeq 9.9\left(1+x^{-1}\right)^{1 / 2}\left(\frac{L_{*}}{L_{\odot}}\right)^{1 / 2}\left(\frac{T_{\mathrm{sub}}}{1300 \mathrm{~K}}\right)^{-2} R_{\odot},
$$




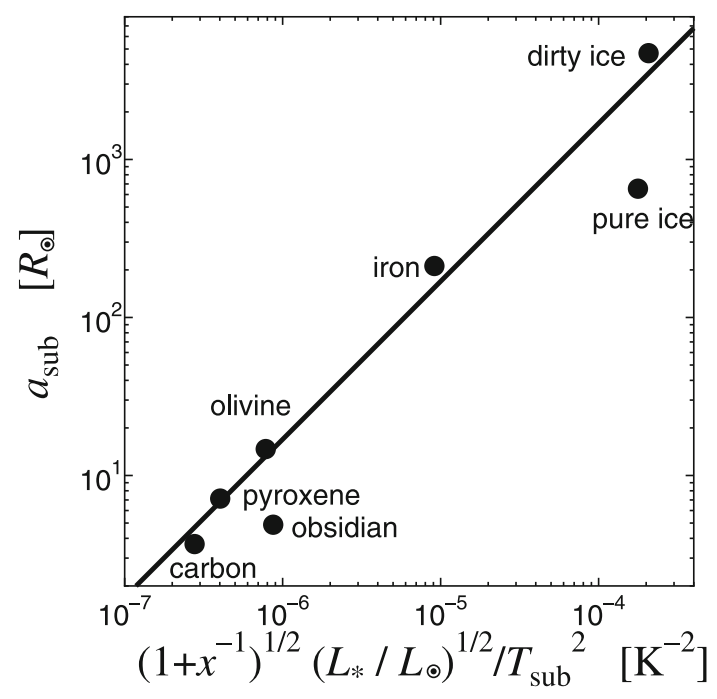

Fig. 2. Sublimation distance $a_{\text {sub }}$ in solar radii $R_{\odot}$ as a function of $\left(1+x^{-1}\right)^{1 / 2}\left(L_{*} / L_{\odot}\right)^{1 / 2} / T_{\text {sub }}^{2}$, where $x$ is given by Eq. (9). The solid line indicates Eq. (13). Circles represent $a_{\text {sub }}$ obtained from the method of Kobayashi et al. (2009) around the sun.

where $R_{\odot}=4.65 \times 10^{-3} \mathrm{AU}$.

Inserting $x$ given by Eq. (9) in Eq. (13), we have $a_{\text {sub }} \propto$ $L_{*}^{1 / 2} T_{\text {sub }}^{-2}$ for $x \gg 1$ and $a_{\text {sub }} \propto M_{*}^{1 / 2} T_{\text {sub }}^{-3}$ for $x \ll 1$. In Kobayashi et al. (2008), our simulations have shown this dependence for dirty ice under the assumption that $L_{*} \propto$ $M_{*}^{3.5}$. We coupled Eqs. (4) and (10) and adopted the cross sections calculated with Mie theory ${ }^{2}$, and then obtained $a_{\text {sub }}$ (Kobayashi et al., 2009). Equation (13) agrees well with $a_{\text {sub }}$ derived from the method of Kobayashi et al. (2009) (see Fig. 2). However, Eq. (13) overestimates $a_{\text {sub }}$ for lessabsorbing materials (pure ice and obsidian) because our assumption of $\bar{C}_{*}=\pi s_{0 \min }^{2}$ is not appropriate for such materials. Nevertheless, Eq. (13) is reasonably accurate for absorbing or compound dust (dirty ice).

\section{Enhancement Factor}

Dust particles with mass $m_{0}$ in the range from $m_{0 \text { min }}$ to $m_{0 \text { max }}$ are mainly controlled by the P-R drag in their source and therefore spiral into the sublimation zone. As mentioned above, the smallest drifting dust with $m_{0 \text { min }}$ corresponds to $\beta=1 / 2$. If the drifting timescale of dust particles due to the P-R drag $t_{\mathrm{PR}}$ is much shorter than the timescale of their mutual, destructive collisions $t_{\mathrm{col}}$, the particles can get out of the dust source region by the P-R drag. The ratio of $t_{\mathrm{PR}}$ to $t_{\text {col }}$ increases with mass or size. Large dust particles with $t_{\mathrm{PR}} \gtrsim t_{\mathrm{col}}$ are collisionally ground down prior to their inward drifts. Therefore, the largest dust $m_{0 \max }$ considered here roughly satisfies the condition $t_{\mathrm{PR}} \sim t_{\mathrm{col}}$ at the source region.

In the steady state, the number density of drifting dust particles is inversely proportional to the drift velocity (e.g.,

\footnotetext{
${ }^{2}$ We apply the complex refractive indices of olivine from Huffman (1976) and Mukai and Koike (1990), of pyroxene from Huffman and Stapp (1971), Hiroi and Takeda (1990), Roush et al. (1991), and Henning and Mutschke (1997), of obsidian from Lamy (1978) and Pollak et al. (1973), of carbon from Hanner (1987), of iron from Johnson and Christy (1974) and Ordal et al. (1988), of ice and dirty ice from Warren (1984) and Li and Greenberg (1997).
}

Kobayashi et al., 2009). Since the drift velocity of dust particles due to the $\mathrm{P}-\mathrm{R}$ drag is proportional to $\beta$, their mass distribution is affected by the mass dependence $\eta=$ $-d \ln \beta / d \ln m$. If the differential mass distribution of the dust source is proportional to $m^{-b}$, that of drifting dust is modulated to $m^{-b+\eta}$ (e.g., Moro-Martín and Malhotra, 2003). Provided that successive collisions mainly produce dust particles in the dust source, we have $b=(11+$ $3 p) /(6+3 p)$ for the steady state of collisional evolution, where $v^{2} / Q_{\mathrm{D}}^{*} \propto m^{-p}$ (Kobayashi and Tanaka, 2010). Here, $Q_{\mathrm{D}}^{*}$ is the specific impact energy threshold for destructive collisions and $v$ is the collisional velocity. From the hydrodynamical simulations and laboratory experiments, $Q_{\mathrm{D}}^{*} \propto$ $m^{-0.2}$ to $m^{0}$ for small dust particles (Holsapple, 1993; Benz and Asphaug, 1999). Since $p=-0.2$ to 0 for a constant $v$ with mass, $b$ is estimated to be $1.8-1.9$. This means that the smallest particles contribute most to the number density before dust particles start to actively sublime, while the largest particles dominate the optical depth prior to active sublimation.

When the temperatures of dust particles reach $T_{\text {sub }}$, they start to sublime actively. Their $\dot{a}$ do not vanish perfectly, but they have very small $|\dot{a}|$ relative to the initial P-R drift velocity. The magnitude of a pile-up due to sublimation is determined by the ratio of these drift rates (Kobayashi et al., 2009). Because the drift rate at the sublimation zone is independent of the initial mass and the initial P-R drift rate decreases with dust mass, the initially small dust piles up effectively. As a result, both the number density and the optical depth at the sublimation zone are determined by the initially smallest dust.

The number density is a quantity that can be measured by in-situ spacecraft instruments, while the optical depth is a key factor for observations by telescopes. In Kobayashi et al. (2009), we have provided enhancement factors for the number density and the optical depth due to sublimation. Here, we apply the simple model for optical cross sections in Eqs. (11), (12) and the properties of the fractal dust given by Eq. (B.7). Furthermore, we take into account an increase of eccentricities from $e_{1}$ due to active sublimation. The number-density enhancement factor $f_{N}$ and the opticaldepth enhancement factor $f_{\tau}$ at the sublimation zone are then given by (see Appendix B for the derivation)

$$
\begin{aligned}
f_{N} & \simeq \frac{b-1-\eta}{b-1} g(x) h\left(e_{1}\right)+1, \\
f_{\tau} & \simeq \frac{2-b}{b-1}\left(\frac{m_{0 \min }}{m_{0 \max }}\right)^{2-b} g(x) h\left(e_{1}\right)+1,
\end{aligned}
$$

where the functions $g(x)$ and $h\left(e_{1}\right)$ include the dependence on $x$ and $e_{1}$, respectively. They are given by

$$
\begin{aligned}
g(x) & =\frac{2 \alpha I(1+x)}{2 \alpha(1+x)+I} \\
h\left(e_{1}\right) & =1-\left[2-\left(2 I e_{1}\right)^{-1 / I}\right]^{(b-1) / \eta}
\end{aligned}
$$

where $\alpha=-d \ln \beta / d \ln s=D-2$ and $I=$ $\mu m_{\mathrm{u}} H / 4 k T_{\text {sub }} \simeq 13$. Since we assume that the mass differential number of the drifting particles is proportional to $m_{0}^{-b+\eta}$ before active sublimation, the dependence of 


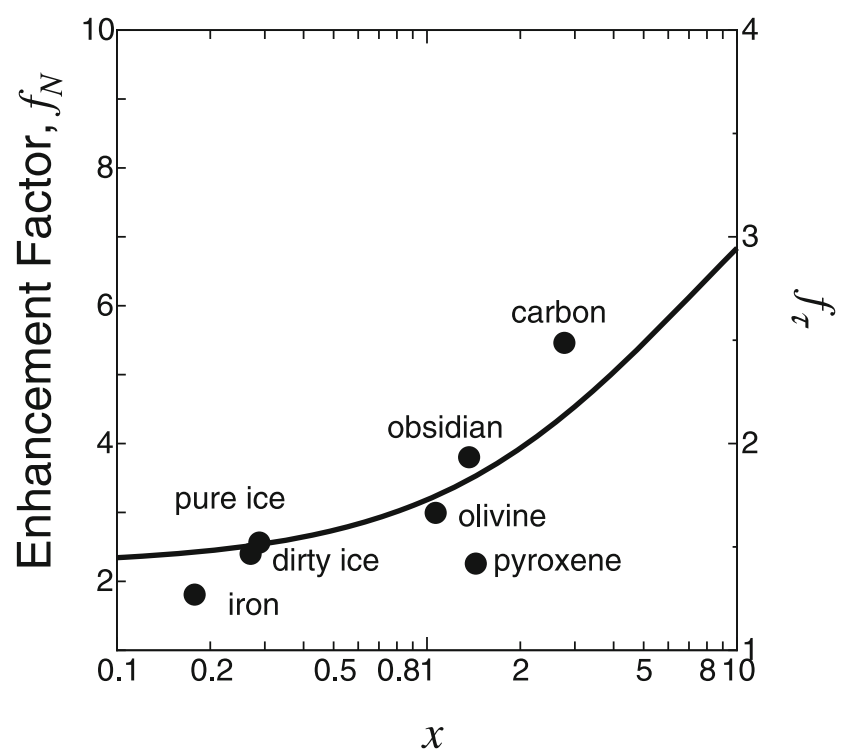

Fig. 3. The enhancement factors for low orbital eccentricities as a function of $x$, where the dimensionless parameter $x$ is determined by Eq. (9). Solid line represents Eqs. (14) and (15) with a use of $m_{0 \min }=m_{0 \max }$ and $h\left(e_{1}\right)=1$. Circles indicate the factor numerically calculated by equations (68) and (69) of Kobayashi et al. (2009) around the sun for spherical dust listed in Table 1.

$f_{\tau}$ on $m_{0 \min } / m_{0 \max }$ seen in Eq. (15) differs from that of Kobayashi et al. (2009). This mass distribution is more realistic and consistent with that of dust particles measured by spacecraft around the earth (Grün et al., 1985). Equation (17) for $h\left(e_{1}\right)$ is applicable for $e_{1}$ ranging from $1 / 2^{I+1} I \simeq 7 \times 10^{-6}$ to $1 / 2 I \simeq 0.05$. Dust particles do not pile up for $e_{1}>1 / 2 I$ and hence we give $h\left(e_{1}\right)=0$ for $e_{1}>1 / 2 I$ (Kobayashi et al., 2009). In addition, $h\left(e_{1}\right)=1$ for $e_{1}<1 / 2^{I+1} I$, while drifting dust particles hardly reach such small eccentricities $\left(e_{1}<1 / 2^{\kappa+1} I \sim 10^{-5}\right)$ because their eccentricities naturally become as high as the ratio of the Keplarian velocity to the speed of light $\left[\sim 10^{-4}(a / 1 \mathrm{AU})^{-1 / 2}\left(M_{*} / M_{\odot}\right)^{1 / 2}\right]$ by the P-R effect.

In Fig. 3, we compare the simplified formulae given by Eqs. (14) and (15) with the enhancement factors rigorously calculated by the formulae of Kobayashi et al. (2009). The $x$ dependence of the enhancement factors given by Eqs. (14), (15) is shown in the function $g(x)$, which is an increasing function ranging from $2 \alpha(x=0)$ to $I(x=\infty)$. Equations (14) and (15) briefly explain the tendency of the enhancement factors; materials with high $x$ produce high enhancement factors.

Because $x$ is proportional to $T_{\text {sub }} / \rho$ (see Eq. (9)), the enhancement factors increase with $T_{\text {sub }} / \rho$. Thus, materials with high sublimation temperature tend to pile up sufficiently. In addition, fluffy dust particles with $D \simeq 2$ cannot effectively pile up even though $\rho$ is low (Kimura et al., 1997). This is explained by the low $\alpha=D-2$ in the function $g(x)$. Particles with $D=3$ produce the highest enhancement factors. In spite of $D=3$, compact particles are not the best for the pile-up due to a high density. Dust particles composed by ballistic particle-cluster aggregation have $D \simeq 3$ but low effective densities relative to compact ones. Therefore, such porous particles with $D \simeq 3$ may

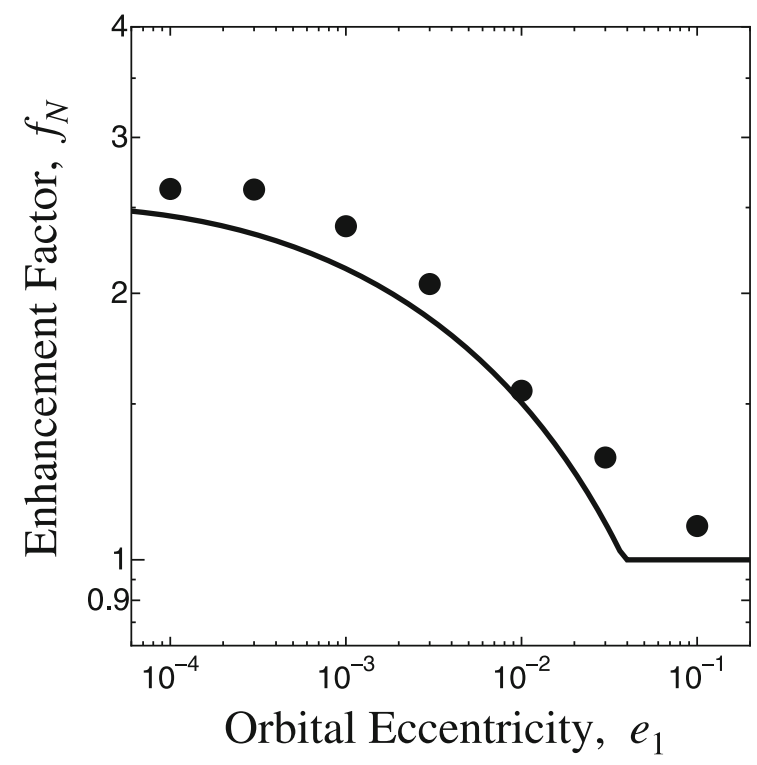

Fig. 4. Dependence of enhancement factor on $e_{1}$ for dirty ice, where $e_{1}$ is orbital eccentricities of dust particles at the beginning of their active sublimation. Solid line indicates Eq. (14). Filled circles represent the results for the simulations calculated by Kobayashi et al. (2008).

produce high enhancement factors due to large $x$ resulting from their low densities. In addition, high $x$ around a luminous star brings the enhancement factors to increase with stellar luminosity, which is shown for dirty ice, obsidian, and carbon in Kobayashi et al. (2008, 2009).

In Kobayashi et al. (2008), we show the eccentricity dependence of enhancement factors from our simulations. The dependence is explained by $h\left(e_{1}\right)$ in the simplified formulae (see Fig. 4). Dust particles can pile up sufficiently for $e_{1} \lesssim 10^{-3}$ because of $h\left(e_{1}\right) \simeq 1$. Otherwise, the enhancement factors decrease with $e_{1}$. For $e_{1} \gtrsim 0.05$, the sublimation ring is not expected.

\section{Recipe}

We briefly show a recipe to obtain the sublimation temperature $T_{\text {sub }}$, its distance $a_{\text {sub }}$, and the enhancement factors $f_{N}, f_{\tau}$. At first, the sublimation temperature $T_{\text {sub }}$ is available from Eq. (5) adopting the material properties $\mu, H$, and $P_{0}$ listed in Table 1 . Then, we calculate the dimensionless parameter $x$ through Eq. (9), applying the stellar luminosity and mass of interest and the bulk density listed in Table 1 for compact spherical dust. Note that we should adopt a lower density for porous particles, taking into account their porosity. Inserting $x$ in Eq. (13), we derive the sublimation distance $a_{\text {sub }}$. We further need orbital eccentricities $e_{1}$ of dust particles at the beginning of active sublimation to calculate the enhancement factors, $f_{N}$ and $f_{\tau}$. The dust particles resulting from collisions have eccentricities $e_{0} \sim \beta$ at the distance $a_{0}$ of the dust production region. Since particles with the highest $\beta$ contribute most to a sublimation ring, we estimate $e_{0} \sim 0.5$. Because eccentricities are dumped by the P-R drag, we can calculate $e_{1}$ from the relation $a_{\mathrm{sub}} e_{1}^{-4 / 5}\left(1-e_{1}^{2}\right)=a_{0} e_{0}^{-4 / 5}\left(1-e_{0}^{2}\right)$ (Wyatt and Whipple, 1950). Inserting $x$ and $e_{1}$ to Eqs. (14) and (15), we obtain $f_{N}$ and $f_{\tau}$. 


\section{Discussion}

The asteroid belt and the Edgeworth-Kuiper belt (EKB) are possible dust sources in the solar system. A dust counter on board spacecraft can measure the number density of dust particles. The sublimation of icy dust occurs at $a_{\text {sub }}=$ $20 \mathrm{AU}$ given by Eq. (13). Icy particles reaching the sublimation zone from the EKB still have high eccentricities $e_{1} \gtrsim 0.1$ (Kobayashi et al., 2008). Therefore, a substantial sublimation ring is unexpected because of $f_{N}=1$ for $h\left(e_{1}\right)=0$. Since the number density of dust particles decreases inside the sublimation zone, only a bump in the radial profile of the number density appears around $a_{\text {sub }}$ (see Kobayashi et al., 2010). In contrast, dust particles composed of rocky, refractory materials actively sublime at several solar radii from the sun. Therefore, orbital eccentricities of dust particles coming from the asteroid belt drop to $\sim 0.01$ around the sublimation distance due to the P-R drag. Since we have $h(0.01) \simeq 0.3$ from Eq. (17) for $b=11 / 6$ and $D=3, f_{N} \simeq 1.3-3.0$ is obtained from Eq. (14) for $x \gtrsim 1$. Therefore, future in-situ measurements of dust could find such a sublimation ring of refractory dust particles originating from the asteroid belt, but not a ring of icy dust particles from the EKB.

A dust ring was observed around $4 R_{\odot}$ from the sun in the period 1966-1983, although it was not detected in the 1990s (Kimura and Mann, 1998 for a review). The optical depth is measured by dust emission observations. The enhancement factor $f_{\tau}$ of the observed ring is estimated to be 2-3 (MacQueen, 1968; Mizutani et al., 1984). The mass distribution of drifting dust particles for $b=11 / 6$ is consistent with the measurement of dust particles with masses ranging from $m_{0 \min } \sim 10^{-12} \mathrm{~g}$ to $m_{0 \max } \sim 10^{-6} \mathrm{~g}$ by spacecraft around the earth orbit (Grün et al., 1985). Using that, we estimate $f_{\tau} \lesssim 1.1$ from Eq. (15) for $e_{1}=0.01$. Thus, the enhancement by sublimation cannot account for the observed dust ring. However, this low $f_{\tau}$ is mainly caused by the mass range of drifting dust particles. If $m_{0 \max }$ decreased during the transport of dust particles from the earth's orbit to the sublimation zone, higher $f_{\tau}$ could be expected. For example, the largest particles in the mass distribution become smaller by the sputtering from the solar wind. The sputtering may decrease $e_{1}$ as well as $m_{0 \max }$. Small particles with high eccentricities from the dust source are ground down by sputtering and blown out by the radiation pressure before reaching the sublimation zone, while large particles with low eccentricities gradually become small by sputtering without the increase of their eccentricities and drift into the sublimation zone. If the ratio of $t_{\mathrm{PR}}$ to the timescale of decreasing size due to sputtering ranges in $0.1-$ 0.7 , sublimation could form such a bright ring because of small $m_{0 \text { max }}$ and $e_{1}$. Indeed, the ratio derived by Mukai and Schwehm (1981) is consistent with the condition for the formation of a sublimation ring. That may be a clue to explain the observed ring.

Debris disks found around main sequence stars would be formed through collisional fragmentation in narrow planetesimal belts, which may resemble the asteroid and Edgeworth-Kuiper belts in the solar system. In young debris disks, fragments produced by successive collisions are removed from the disk by radiation pressure. We call such a disk a collision-dominated disk. Once the amount of bodies has significantly been decreased through this process, the P-R drag becomes the main removal process of fragments. Such a disk is referred to as a drag-dominated disk. We have investigated the dust ring formation in drag-dominated disks. To observe a sublimation ring requires a high enhancement factor $f_{\tau}$ for the optical depth. As shown in Eq. (15), a small ratio of $m_{0 \max }$ to $m_{0 \text { min }}$ yields high $f_{\tau}$. The condition of $m_{0 \max } \sim m_{0 \min }$ is expected to form a bright ring. Since the drift time due to the P-R drag is comparable to the collisional time for bodies with $m_{0 \max }$, the condition of $m_{0 \max } \sim m_{0 \text { min }}$ is achieved in transition from a collisiondominated disk to a drag-dominated one.

A significant sublimation ring consisting of icy particles is not expected in a debris disk due to high eccentricities if a planetesimal belt as a dust source is located within a few hundreds AU, similar to the solar system. On the contrary, dust particles composed of refractory materials have $e_{1} \sim 0.01$ or smaller if a planetesimal belt is around the distance of the asteroid belt or further outside. Furthermore, refractory dust particles have high sublimation temperatures and hence produce a higher enhancement factor. Recently, inner debris disks of refractory grains have been observed through interferometry around Vega, $\tau$ Cet, $\zeta$ Aql, and $\beta$ Leo, and Formalhaut (Absil et al., 2006, 2008; Di Folco et al., 2007; Akeson et al., 2009). Such inner debris disks may have notable sublimation rings.

To check the observability of a sublimation ring in the spectral energy distribution (SED) of thermal emission expected from a disk around Vega located at the distance of $7.6 \mathrm{pc}$ from the earth, we take our formulae with $M_{*}=$ $2.1 M_{\odot}$ and $L_{*}=59 L_{\odot}$ for compact spherical olivine particles. We obtain $T_{\text {sub }}=1300 \mathrm{~K}, a_{\text {sub }}=0.35 \mathrm{AU}$ and $f_{\tau}=3.1$, where we adopt $m_{0 \max }=m_{0 \min }$ and $h\left(e_{1}\right)=1$ in Eq. (15). The smallest radius $s_{0 \mathrm{~min}}=10 \mu \mathrm{m}$ is much larger than the peak wavelength of thermal emission $\left(\lambda_{\text {sub }} \simeq\right.$ $2.3 \mu \mathrm{m})$ and hence we simply treat dust particles as blackbodies to calculate the SED. The optical depth $\tau_{\mathrm{d}}$ of dust particles drifting by the P-R drag without sublimation from the outer edge $a_{\text {out }}$ to the inner one $a_{\text {in }}$ is given by a con-

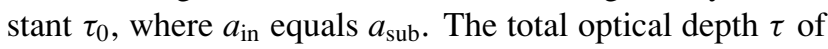
the disk can be set as

$$
\begin{aligned}
\tau & =\tau_{\mathrm{d}}+\tau_{\mathrm{e}}, \\
\tau_{\mathrm{d}} & = \begin{cases}\tau_{0} & \text { for } a_{\text {in }}<r<a_{\text {out }}, \\
0 & \text { otherwise, }\end{cases} \\
\tau_{\mathrm{e}} & = \begin{cases}\tau_{0}\left(f_{\tau}-1\right)\left(a_{\text {sub }}+\delta a_{\text {sub }}-r\right) / \delta a_{\text {sub }} \\
\text { for } a_{\text {sub }}<r<a_{\text {sub }}+\delta a_{\text {sub }},\end{cases}
\end{aligned}
$$

where $\tau_{\mathrm{e}}$ denotes the optical depth of dust particles forming a dust ring with width $\delta a_{\text {aub }}$. The width of the ring is roughly given by $\delta a_{\text {sub }}=0.05 a_{\text {sub }}$ (Kobayashi et al., $2008,2009)$. We set $a_{\text {out }}=1.5 a_{\text {sub }}$. Note that the other choice of $a_{\text {out }}$ does not change our result drastically. Choosing $\tau_{0}=8 \times 10^{-3}$, we can reproduce the flux density $\simeq 8.7 \mathrm{Jy}$ measured by Absil et al. (2006) with the interferometry at a wavelength of $2.1 \mu \mathrm{m} .{ }^{3}$ Figure 5 depicts that 


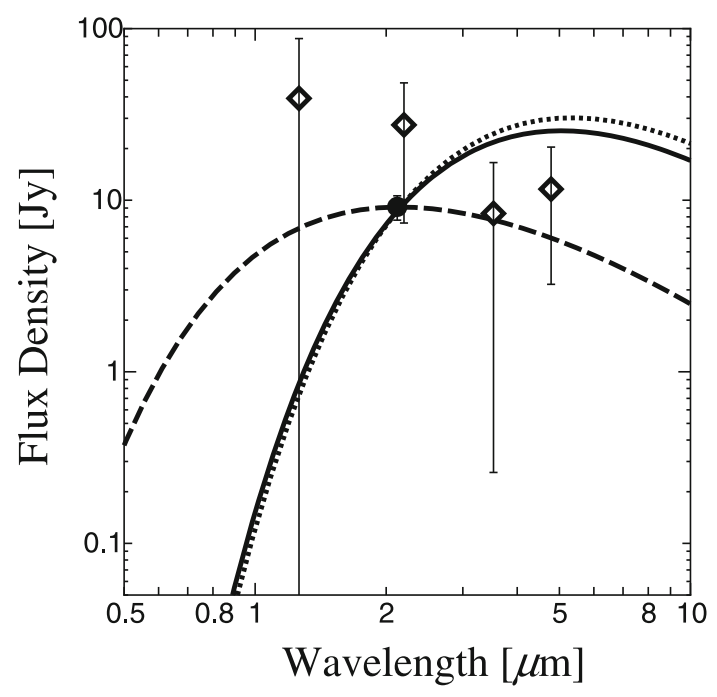

Fig. 5. The spectral density distribution of the disk around Vega. The solid line indicates the flux density from a disk with the sublimation ring, choosing $\tau_{0}=8 \times 10^{-3}$ to fit the observational data at $2.1 \mu \mathrm{m}$. Dotted lines represent that without a sublimation ring $\left(f_{\tau}=1\right.$ resulting in $\tau_{\mathrm{e}}=0$ ) for $\tau_{0}=1 \times 10^{-2}$. To check the $a_{\text {sub }}$ dependence, we set $a_{\text {in }}=0.6 a_{\text {sub }}$ for $\tau_{0}=4 \times 10^{-4}$ in the case without the sublimation ring (dashed lines). Circle corresponds to the interferometric measurement by Absil et al. (2006) and diamonds indicate the photometric data (Absil et al., 2006 and reference therein).

the sublimation ring does not bring about a noticeable spectral feature in the SED, while the SED strongly depends on $a_{\text {sub }}$ value. The flux density from the disk diminishes with decreasing wavelength for the wavelength smaller than $\lambda_{\text {sub }}$ because of the absence of dust particles with temperature higher than $T_{\text {sub }}$ due to sublimation. The result for $a_{\text {in }}=0.6 a_{\text {sub }} \simeq 0.21 \mathrm{AU}$ is shown to better agree with the observational data. Since $a_{\text {sub }} \simeq 0.22 \mathrm{AU}\left(T_{\text {sub }} \simeq 1700 \mathrm{~K}\right)$ for pyroxene, the Vega disk seems to be abundant in pyroxene compared to olivine. That could be recognized as an evidence of sublimation unless the light scattering of dust particles exceed their thermal emission. ${ }^{4}$

\section{Summary}

1. We provide formulae of enhancement factors for the number density and the optical depth due to a pile-up of dust particles caused by sublimation and its location and sublimation temperature, applying a simple model for optical cross sections of fractal dust particles.

2. High sublimation temperatures result in substantial enhancement factors, though the pile-up is insensitive to the optical properties of dust particles.

\footnotetext{
${ }^{3}$ Note that $\tau_{0}$ depends on the collision and drift timescales, $t_{\mathrm{c}}, t_{\mathrm{PR}}$, in a planetesimal disk. Drag-dominated disks should satisfy the condition $t_{\mathrm{c}} \gtrsim t_{\mathrm{PR}} ; \tau_{0} \lesssim(1+\gamma) v_{\mathrm{k}} / c$ with the Keplarian velocity $v_{\mathrm{k}}$ and the ratio $\gamma$ of the P-R drag force due to the stellar wind to that due to the stellar radiation. The value of $\tau_{0}$ applied for fitting is much larger than that for the drag-dominated disk ( $\tau_{0} \lesssim 3 \times 10^{-4}$ ), if we only consider the P-R drag by the stellar radiation. However, the mass loss rate of Vega is estimated to be less than $3.4 \times 10^{-10} M_{\odot} \mathrm{yr}^{-1}$ from radio-continuum observations (Hollis et al., 1985). For the upper limit of the mass loss rate, drag-dominated disks can have the optical depth $\tau_{0} \lesssim 7 \times 10^{-2}$ because of the P-R drag due to the stellar wind $(\gamma \sim 300)$. Thus the disk around Vega may be a drag-dominated disk.

${ }^{4}$ The scattering of light from the disk around Vega is negligible around the wavelength $\sim 1 \mu \mathrm{m}$ (Absil et al., 2006).
}

3. If we adopt the mass distribution measured around the earth, the enhancement factor for the optical depth near the sun is smaller than 1.1. Therefore, the enhancement cannot explain the solar dust ring detected in the epoch of 1966-1983, unless the largest particles were destroyed by sputtering.

4. The number-density enhancement factor is expected to be 1.4-3 in the vicinity of the sun. Therefore, a sublimation ring could be found by in-situ measurements by spacecraft around several solar radii from the sun.

5. Sublimation removes dust particles with temperatures higher than their sublimation temperature. In the spectral energy distribution, the flux density from a disk reduces with decreasing wavelength, if the wavelength is shorter than the peak one of the blackbody spectrum with the sublimation temperature. That could be seen as a sublimation evidence. However, it is difficult to find signs from a sublimation ring in the spectral energy distribution.

Acknowledgments. We appreciate the advice and encouragement of A. Krivov, M. Ilgner, and M. Reidemeister. The careful reading of the manuscript by the anonymous reviewers helps its improvement. This research is supported by grants from CPS, JSPS, and MEXT Japan.

\section{Appendix A. Application Limit}

The value of $\beta$ increases with decreasing radius as long as the radius fulfills the condition $s \gtrsim \lambda_{*}$. For $s \lesssim \lambda_{*}$, however, it decreases with decreasing radius. ${ }^{5}$ Hence, $\beta$ has a maximum value at $s \sim \lambda_{*}=\left(2898 \mathrm{~K} / T_{*}\right) \mu \mathrm{m}$. From Eq. (2), the maximum value of $\beta$ is approximately given by

$$
\begin{aligned}
\beta_{\max } \sim & 0.98\left(\frac{L_{*}}{L_{\odot}}\right)\left(\frac{M_{\odot}}{M_{*}}\right) \\
& \times\left(\frac{T_{*}}{5 \times 10^{3} \mathrm{~K}}\right)\left(\frac{\rho}{1.0 \mathrm{~g} \mathrm{~cm}^{-3}}\right)^{-1},
\end{aligned}
$$

where the radiation pressure cross section averaged over the stellar radiation spectrum $\bar{C}_{\mathrm{pr}} \sim \pi s^{2}$ for $s \gtrsim \lambda_{*}$. Here we define the effective density by

$$
\rho=\frac{3 m}{4 \pi s^{3}}
$$

with the use of the dust mass $m$ and the characteristic radius $s$. Note that $\rho$ depends on $s$ in general; $\rho$ is constant for $m \propto s^{3}$ (e.g., a compact sphere), whereas $\rho$ is proportional to $s^{D-3}$ for a fractal aggregate with fractal dimension $D$ (see Mukai et al., 1992 for the relation).

When dust particles are produced by successive collisions between large bodies, their largest $\beta$ does not exceed $1 / 2$ because the dust with $\beta>1 / 2$ cannot resist against strong radiation pressure. These particles then drift into the sublimation zone. The smallest drifting dust particles that have the largest $\beta$ contribute most to the pile-up caused by sublimation. Because the enhancement factors of the number density and the optical depth are proportional to the largest $\beta$ value that drifting dust particles attain, we may

\footnotetext{
${ }^{5}$ Note that $\beta$ is independent of $s$ for much smaller particles (Gustafson,
} 1994). 
expect insufficient pile-ups of the particles if $\beta_{\max }<1 / 2$. We thus derive simplified formulae for characteristics of a dust ring from the assumption of $\beta_{\max }>1 / 2$. Because the variation of $L_{*}$ is much larger than that of $T_{*}$ for main sequence stars, this assumption is translated into the condition for stellar luminosity given by

$$
\frac{L_{*}}{L_{\odot}} \gtrsim 0.5\left(\frac{M_{*}}{M_{\odot}}\right)\left(\frac{T_{*}}{5 \times 10^{3} \mathrm{~K}}\right)^{-1}\left(\frac{\rho}{1.0 \mathrm{~g} \mathrm{~cm}^{-3}}\right),
$$

if $\bar{C}_{\mathrm{pr}} \simeq \pi s^{2}$ for $s \gtrsim \lambda_{*}$.

\section{Appendix B. Derivation of Enhancement Factors}

We assume that the mass differential number of drifting dust particles is proportional to $m_{0}^{-b+\eta}$ for the drifting particles with mass $m_{0}$. In addition, we adopt $\beta(m) \propto m^{-\eta}$, and $S(m) \propto m^{\zeta s}$, where $S$ is the geometrical cross section of a dust particle with mass $m$. In Kobayashi et al. (2009), we derived the number-density enhancement factor $f_{N}$ and the optical-depth enhancement factor $f_{\tau}$ at the peak as

$$
\begin{aligned}
& f_{N}=\eta\left(m_{0 \min }\right) g_{\mathrm{m}}\left(T_{\mathrm{sub}}, m_{0 \min }\right) h_{1}\left(y_{1}, y_{2}\right)+1 \text {, } \\
& f_{\tau}=\eta\left(m_{0 \min }\right) g_{\mathrm{m}}\left(T_{\mathrm{sub}}, m_{0 \min }\right) h_{2}\left(y_{1}, y_{2}\right)+1 \text {, }
\end{aligned}
$$

where

$$
\begin{aligned}
& h_{1}\left(y_{1}, y_{2}\right)=\frac{\int_{1}^{y_{1}} \tilde{y}^{-b} d \tilde{y}}{\int_{1}^{y_{2}} \tilde{y}^{-b+\eta} d \tilde{y}}, \\
& h_{2}\left(y_{1}, y_{2}\right)=\frac{\int_{1}^{y_{1}} \tilde{y}^{-b} d \tilde{y}}{\int_{1}^{y_{2}} \tilde{y}^{-b+\eta+\zeta_{S}} d \tilde{y}},
\end{aligned}
$$

$y_{1}=m_{\text {init,max }} / m_{0 \min }$, and $y_{2}=m_{0 \max } / m_{0 \min }$. As we will describe below, dust particles with $m_{0}<m_{\text {init,max }}$ drifting into the sublimation zone can contribute to the enhancement factors.

Here $g_{\mathrm{m}}$ is a function of the optical properties of dust particles with $m=m_{0 \min }$ at $T_{\text {sub }}$, namely, given by a function of $x$. The function $g_{\mathrm{m}}$ is defined as

$g_{\mathrm{m}}(T, m)=\frac{\frac{2}{4+c_{T}}\left(\frac{d \ln P_{\mathrm{v}}}{d \ln T}-\frac{1}{2}\right)-2}{1+\frac{\eta}{1-\beta}-\eta-\zeta_{A}-\frac{d \ln \eta}{d \ln m}-\left(\frac{d \ln P_{\mathrm{v}}}{d \ln T}-\frac{1}{2}\right) \frac{c_{*}-c_{\mathrm{d}}}{4+c_{T}}}$,

where

$$
\begin{gathered}
c_{*}=\frac{d \ln \bar{C}_{*}}{d \ln m}, \quad c_{\mathrm{d}}=\left(\frac{\partial \ln \bar{C}_{\mathrm{d}}}{\partial \ln m}\right)_{T}, \\
c_{T}=\left(\frac{\partial \ln \bar{C}_{\mathrm{d}}}{\partial \ln T}\right)_{m}, \quad \zeta_{A}=\frac{d \ln A}{d \ln m} .
\end{gathered}
$$

According to our simple model in Sections 2 and 3, a particle with $T_{\text {sub }}$ and $m_{0 \text { min }}$ has

$c_{*}=2 / D, \quad c_{\mathrm{d}}=2 / D+1 /(x+1) D, \quad \zeta_{A}=\zeta_{S}=2 / D$, $\eta=-d \ln \beta / d \ln m=(D-2) / D, \quad \beta=1 / 2$.

${ }^{6}$ Equation (B.10) is different from equation (57) in Kobayashi et al. (2009) because $d \ln T / d \ln a$ in their equation (29) should be replaced by $\partial \ln T / \partial \ln a$. Then, we obtain Eq. (B.10) instead of their equation (57).
Then, $g\left(T_{\text {sub }}, m_{0 \min }\right)$ reduces to

$$
g_{\mathrm{m}}\left(T_{\mathrm{sub}}, m_{0 \min }\right)=\frac{2 I D(1+x)}{2 \alpha(1+x)+I}
$$

where $c_{T}=0$, and $4 I=d \ln P_{\mathrm{v}} / d \ln T \gg 1$. We define $\eta g_{\mathrm{m}}\left(T_{\text {sub }}, m_{0 \mathrm{~min}}\right)$ as $g(x)$ given by Eq. (16).

Only the dust particles with initial masses ranging from $m_{0 \text { min }}$ to $m_{\text {init,max }}$ can stay long around the distance $a_{\text {sub }}$ for a pile-up. Large particles initially pass the distance $a_{\text {sub }}$ and approach there again by outward drift due to active sublimation (see figure 1 of Kobayashi et al., 2008). Orbital eccentricities $e$ of the particles rise during the active sublimation. If $e \gtrsim 2 k T_{\text {sub }} / \mu m_{\mathrm{u}} H$, they are blown out immediately and hence do not contribute to the formation of a dust ring. Therefore, $m_{\text {init,max }}$ depends on the eccentricity $e_{1}$ of a dust particle starting the active sublimation. Kobayashi $e t$ $a l$. (2009) derive the relation between $e$ and $m$ during the active sublimation as

$$
e=\left(\frac{1-\beta\left(m_{1}\right)}{1-\beta(m)}\right)^{\kappa} e_{1}
$$

where

$$
\kappa=\frac{1}{4+c_{T}}\left(\frac{d \ln P_{\mathrm{v}}}{d \ln T}-\frac{1}{2}\right)-\frac{5}{4},
$$

with $m_{1}$ is the mass starting the active sublimation. ${ }^{6}$ We approximate $\kappa=I$ because $I \gg 1$. Equation (B.9) indicates that $e$ substantially changes for $\beta \sim 1$. Since the mass loss is negligible outside the sublimation zone, we approximate $m_{1} \simeq m_{0}$. Particles can pile up as long as $e \lesssim$ $2 k T_{\text {sub }} / \mu m_{\mathrm{u}} H=1 / 2 I$ (Kobayashi et al., 2009). Substituting $e=1 / 2 I, \beta=1 / 2$, and $\beta_{1}=\beta\left(m_{\text {init, } \max } / m_{0 \text { min }}\right)^{-\eta}$ into Eq. (B.9), we have

$$
m_{\text {init,max }}=\left[\frac{\left(2 I e_{1}\right)^{1 / I}}{2\left(2 I e_{1}\right)^{1 / I}-1}\right]^{1 / \eta} m_{0 \min } .
$$

Equation (B.11) is valid for $m_{\text {init,max }} \leq m_{0 \max }$ and $e_{1} \geq$ $1 / I 2^{I+1}$. We should set $m_{\text {init,max }}=m_{0 \max }$ instead of Eq. (B.11) for $m_{\text {init, } \max }>m_{0 \max }$ or $e_{1}<1 / I 2^{I+1}$. Because $y_{2} \gg 1$, and $b=1.8-1.9$ in Eqs (B.3) and (B.4), $h_{1}$ and $h_{2}$ are given by

$$
\begin{aligned}
& h_{1}\left(y_{1}, y_{2}\right)=\frac{b-\eta-1}{b-1} h\left(e_{1}\right), \\
& h_{2}\left(y_{1}, y_{2}\right)=\frac{2-b}{b-1}\left(\frac{m_{0 \min }}{m_{0 \max }}\right)^{2-b} h\left(e_{1}\right),
\end{aligned}
$$

where $h$ is defined as Eq. (17).

Substituting Eqs. (B.8), (B.12), and (B.13) into Eqs. (B.1) and (B.2), we have the enhancement factors in Eqs. (14) and (15).

\section{References}

Absil, O. et al., Circumstellar material in the Vega inner system revealed by CHARA/FLUOR, Astron. Astrophys., 452, 237-244, 2006.

Absil, O. et al., A near-infrared interferometric survey of debris disc stars. II. CHARA/FLUOR observations of six early-type dwarfs, Astron. Astrophys., 487, 1041-1054, 2008.

Akeson, R. L. et al., Dust in the inner regions of debris disks around a stars, Astrophys. J., 691,1896-1908, 2009. 
Belton, M. J. S., Dynamics of interplanetary dust, Science, 151, 35-44, 1966.

Benz, W. and E. Asphaug, Catastrophic disruptions revisited, Icarus, 142, 5-20, 1999.

Burns, J. A., P. L. Lamy, and S. Soter, Radiation forces on small particles in the solar system, Icarus, 40, 1-48, 1979.

Di Folco, E. et al., A near-infrared interferometric survey of debris disk stars. I. Probing the hot dust content around $\epsilon$ Eridani and $\tau$ Ceti with CHARA/FLUOR, Astron. Astrophys., 475, 243-250, 2007.

Grigorieva, A., Ph. Thébaut, P. Artymowicz, and A. Brndeker, Survival of icy grains in debris discs The role of photosputtering, Astron. Astrophys., 475, 755-764, 2007.

Grün, E., H. A. Zook, H. Fechtig, and R. H. Giese, Collisional balance of the meteoritic complex, Icarus, 62, 244-272, 1985.

Gustafson, B. A. S., Physics of zodiacal dust, Ann. Rev. Earth Planet. Sci., 22, 553-595, 1994

Hanner, M., Grain optical properties, in Infrared Observations of Comets Halley \& Wilson and Properties of the Grains, edited by M. Hanner, pp. 22-49, NASA Conference, Washington, 1987.

Henning, T. and H. Mutschke, Low-temperature infrared properties of cosmic dust analogues, Astron. Astrophys., 327, 743-754, 1997.

Hiroi, T. and H. Takeda, A method to determine silicate abundances from reflectance spectra with applications to Asteroid 29 amphitrite associating it with primitive achondrite meteorites, Icarus, 88, 205-227, 1990.

Hollis, J. M., G. Chin, and R. L. Brown, An attempt to detect mass loss from Alpha Lyrae with the VLA, Astrophys. J., 294, 646-648, 1985.

Holsapple, K. A., The scaling of impact processes in planetary sciences, Ann. Rev. Earth Planet. Sci., 21, 333-373, 1993.

Huffman, D. R., Optical properties of particulates, Astrophys. Space Sci. Libr., 55, 191-200, 1976.

Huffman, D. R. and J. L. Stapp, Interstellar dust-Silicate extinction related to the $2200 \AA$ A band, Nature, 229, 45-46, 1971.

Johnson, P. B. and R. W. Christy, Optical constants of transition metals: Ti, V, Cr, Mn, Fe, Co, Ni, and Pd, Phys. Rev. B, 9, 5056-5070, 1974.

Kimura, H. and I. Mann, Brightness of the solar F-corona, Earth Planets Space, 50, 493-499, 1998.

Kimura, H., H. Ishimoto, and T. Mukai, A study on solar dust ring formation based on fractal dust models, Astron. Astrophys., 326, 263-270, 1997.

Kobayashi, H. and H. Tanaka, Fragmentation model dependence of collision cascades, Icarus, 206, 735-746, 2010.

Kobayashi, H., S. Watanabe, H. Kimura, and T. Yamamoto, Dust ring formation due to ice sublimation of radially drifting dust particles under the Poynting Robertson effect in debris disks, Icarus, 195, 871-881, 2008.

Kobayashi, H., S. Watanabe, H. Kimura, and T. Yamamoto, Dust ring formation due to sublimation of dust grains drifting radially inward by the Poynting-Robertson drag: An analytical model, Icarus, 201, 395405, 2009.

Kobayashi, H., H. Kimura, S. Yamamoto, S. Watanabe, and T. Yamamoto, Ice sublimation of dust particles and their detection in the outer solar system, Earth Planets Space, 62, 57-61, 2010.

Lamy, P. L., Interaction of interplanetary dust grains with the solar radiation field, Astron. Astrophys., 35, 197-207, 1974.

Lamy, P. L., Optical properties of silicates in the far ultraviolet, Icarus, 34, 68-75, 1978.

Li, A. and J. M. Greenberg, A unified model of interstellar dust, Astron. Astrophys., 323, 566-584, 1997.

MacQueen, R. M., Infrared observations of the outer solar corona, Astrophys. J., 154, 1059-1976, 1968.

Mizutani, K., T. Maihara, H. Takami, and N. Hiromoto, Near-infrared observation of the circumsolar dust emission during the 1983 solar eclipse, Nature, 312, 134-136, 1984.

Moro-Martín, A. and R. Malhotra, Dynamical models of Kuiper belt dust in the inner and outer solar system, Astron. J., 125, 2255-2265, 2003.

Mukai, T. and C. Koike, Optical constants of olivine particles between wavelengths of 7 and 200 microns, Icarus, 87, 180-187, 1990.

Mukai, T. and G. Schwehm, Interaction of grains with the solar energetic particles, Astron. Astrophys., 95, 373-382, 1981.

Mukai, T. and T. Yamamoto, A model of the circumsolar dust cloud, Publ. Astron. Soc. Jpn., 31, 585-596, 1979.

Mukai, T., H. Ishimoto, T. Kozasa, J. Blum, and J. M. Greenberg, Radiation pressure forces of fluffy porous grains, Astron. Astrophys., 262, 315320, 1992.

Ordal, M. A., R. J. Bell, R. W. Alexander, Jr., L. A. Newquist, and M. R. Querry, Optical properties of $\mathrm{Al}, \mathrm{Fe}, \mathrm{Ti}, \mathrm{Ta}, \mathrm{W}$, and Mo at submillimeter wavelengths, Appl. Opt., 27, 1203-1209, 1988.

Pollack, J. B., O. B. Toon, and B. N. Khare, Optical properties of some terrestrial rocks and glasses, Icarus, 19, 372-389, 1973.

Roush, T., J. Pollack, and J. Orenberg, Derivation of midinfrared (5-25 microns) optical constants of some silicates and palagonite, Icarus, 94, 191-208, 1991.

Warren, S. G., Optical constants of ice from the ultraviolet to the microwave, Appl. Opt., 23, 1206-1225, 1984.

Washburn, E. W., International Critical Tables of Numerical Data Physics, Chemistry and Technoloty (Vol. III), McGraw-Hill Book Company, Inc. New York and London, 1928.

Wyatt, S. P. and F. L. Whipple, The Poynting-Robertson effect on meteor orbits, Astrophys. J., 111, 134-141, 1950.

H. Kobayashi (e-mail: hkobayas@astro.uni-jena.de), H. Kimura, S.-i. Watanabe, T. Yamamoto, and S. Müller 